\title{
IMAGINARIO POLÍTICOY DISCURSOS TÉCNICOS EN LAS REFORMAS ADMINISTRATIVAS. LOS CASOS DE ARGENTINA, CHILE Y URUGUAY EN LA DÉCADA DE 1990
}

\section{THE POLITICAL IMAGINARY AND TECHNICAL DISCOURSE IN ADMINISTRATIVE REFORMS. THE ARGENTINA, CHILE AND URUGUAY CASE IN THE 1990s}

\author{
MARCELO MORICONI BEZERRA \\ ISCTE - Instituto Universitario de Lisboa, CIES-IUL, Portugal \\ Marcelo.moriconi@iscte.pt
}

\section{RESUMEN}

El trabajo analiza los imaginarios políticos en los discursos oficiales en torno a las reformas de la administración pública en Argentina, Chile y Uruguay, en la década del noventa. Los conceptos técnicos en los que se basan los nuevos modos de gestión no significan por sí mismos y dependen de sistemas de ideas más amplios, que incluyen el significado de la democracia y del Estado, sedimentado en cada caso particular. Conceptos derivados de la escuela del análisis de discursos, como hegemonía, puntos nodales y sedimentación, permiten ver las estrategias de construcción de la realidad simbólica y determinar lo hacible y tolerable en cada cultura política. La elección de realidades limitadas, resumidas a las instituciones formales, es una constante en los casos analizados, lo que explica por qué las reformas fueron ineficaces para eliminar prácticas culturales habituales como el clientelismo.

Palabras clave: Discurso, Reformas administrativas, Imaginarios políticos, América Latina.

\begin{abstract}
This article analyzes the political imaginaries in the official discourses about the public administration reforms in Argentina, Chile and Uruguay, during the nineties. The new management methods are based on technical concepts that do not signify by themselves. To acquire effective meaning, these concepts depend on systems of ideas about the significance of the State and democracy, settled in each particular case. Concepts derived from the discourse analysis school, such as hegemony, nodal points or sedimentation, are useful to understand the strategies to construct the symbolic reality in each case. This reality will depend on the political imaginary which determines 'what is possible to do' and 'what is tolerable' in each particular society. The election of limited realities, focused only on formal institutions, has been constant in the analyzed discourses, which explains why the reforms were inefficient to remove common cultural practices such as clientelism.
\end{abstract}

Keywords: Discourses, Administrative reforms, Political imaginaries, Latin America.

Revista de Gestión Pública

VOLUMEN I, Número 1

ISSN 0719-1820

pp. 7-36 


\section{INTRODUCCIÓN}

Cerca de dos décadas han transcurrido desde que los países latinoamericanos incorporaron a sus agendas cuestiones destinadas a reformar, al menos desde lo discursivo, la situación de la administración pública. A pesar de la abundante bibliografía (Przeworski 1991, Geddes 1994, Acuña y Smith 1994, Oszlak 2001, Haggard y Kaufman 1992), las administraciones públicas de la región no han mejorado sustancialmente y se mantienen vicios culturales y políticos como el clientelismo, la corrupción, y el nombramiento por intereses o preferencias personales, sin base en el mérito (Ozslak 2001). Estos problemas no se relacionan con la falta de legislación sino con su incumplimiento (Ferraro 2006).

En este sentido, el artículo compara los discursos oficiales ${ }^{1}$ de los gobiernos de Carlos Menem, (Argentina 1990-1999), Eduardo Frei RuízTagle (Chile 1994-2000), y Julio María Sanguinetti (Uruguay 1995-2000) para determinar el marco narrativo bajo el cual se impulsaron las primeras reformas globales de la gestión pública en cada país analizado. Para esta tarea se retoman conceptos clave de la escuela del análisis de discurso como hegemonía, dislocación, (Laclau y Mouffe 1985), puntos nodales (Zizek 1992), ideología (Fairclough 1989, 1992).

El rol de las ideas en la construcción de los marcos de interpretación de coyuntura desde los cuales la clase política actúa y diagnostica focos de conflicto sobre los cuales actuar ha sido pasado por alto, como objeto de estudio, en la mayor parte de la literatura sobre las reformas administrativas (Panizza 2002). Francisco Panizza $(2002,2004)$ planeta la necesidad de determinar cómo lo que era impensable tiempo atrás se ha convertido en la actualidad en el nuevo sentido común. Hacerse estas preguntas implica partir de una concepción constructivista del orden social y concebir a los lenguajes, las comunidades y las instituciones como producto del tiempo y las circunstancias, y no de una lógica o principio fundamental.

Comprender cómo los gobiernos diagnostican la coyuntura para legitimar los cambios impulsados y analizar el valor semántico con el que se resignifican los conceptos técnicos que se incorporan al discurso como herramientas y valores finalistas permite ver la complejidad del juego político a la hora de establecer cambios que afecten al diseño institucional y aceptar las modificaciones.

\footnotetext{
Cabe aclarar que el discurso no se resume al lenguaje, sea hablado o escrito. En rasgos generales, los discursos serán entendidos como "toda acción portadora de sentido" (Laclau 2002). En este sentido se analizan discursos oficiales, programas de gobierno, leyes y decretos.
} 


\section{LLEGADA AL PODER: CONTEXTO HISTÓRICO Y ESTRATEGIA DE ENUNCIACIÓN}

Los tres gobiernos analizados iniciaron sus gestiones en coyunturas muy diferentes. En el caso argentino, Carlos Menem asumió la presidencia antes de tiempo, tras la renuncia de Raúl Alfonsín. El contexto social y económico era de una gravedad absoluta, con picos hiperinflacionarios del $5.000 \%{ }^{2}$.

Mientras tanto, en Uruguay la situación era más calmada. Si bien el discurso sobre la necesidad de reformas había comenzado en la primera gestión democrática tras la dictadura (Julio María Sanguinetti, 1985-1990), y se había sedimentado durante la gestión de Luis Lacalle (1990-1995), fue durante el segundo mandato de Julio María Sanguinetti cuando se presentó por primera vez un plan general de modernización de la administración pública. La coyuntura en la que se impulsó el plan fue muy distinta a la de Argentina. Si bien la economía uruguaya no era un éxito debido a la crisis internacional (Efecto Tequila), no existía un contexto hiperinflacionario. Por otra parte, durante la gestión anterior el tema de la reforma se había incorporado a la agenda pública y la posibilidad de proceder a impulsar medidas de corte neoliberal había sufrido un revés importante en el referéndum que detuvo el proceso privatizador ${ }^{3}$.

En Chile, la reforma general se impulsó durante la gestión de Eduardo Frei Ruíz-Tagle, si bien hay que tener en cuenta que el país fue pionero en imponer una reorganización de corte neoliberal con amplia apertura de mercado durante la dictadura. En la década del setenta, el pinochetismo impulsó un cambio paradigmático ${ }^{4}$ (Hall 1986) por coerción, que

\footnotetext{
2 Antes de este pico hiperinflacionario, la inflación anual promedio desde el retorno de la democracia había sido $343,56 \%$.

3 El 13 de diciembre de 1993 se realizó un referéndum para que la ciudadanía decidiera el apoyo o no a la Ley de Empresas Públicas, la principal propuesta del gobierno de Lacalle, que dejaba abierta la posibilidad de privatizar las empresas estatales. El $72 \%$ de los votantes se opuso a las privatizaciones.

4 Hall sostiene que el proceso de formulación de políticas envuelve habitualmente tres variables centrales: los objetivos fundamentales que guían la política, las técnicas utilizadas para alcanzar esas metas y el entorno preciso de esos instrumentos. Estos cambios son clasificados como de tercer, segundo y primer orden. "Los cambios de primer orden acontecen en períodos en los cuales el paradigma político dominante opera 'normalemente' y los cambios son concebidos esencialmente en términos técnicos. En tanto, los cambios de tercer orden ocurren cuando los basamentos, objetivos y fines de la política son reformulados, resultando en un 'cambio de paradigma'" (Panizza 2002: 66-67).
} 
incluyó recortes de personal en la administración, privatizaciones, apertura de mercado y descentralizaciones 5 . Guiada por técnicos de la Escuela de Chicago, cuna de la economía neoclásica, las reformas durante la dictadura chilena fueron el primer experimento de este tipo a nivel mundial. Tras la reinstauración de la democracia, mediante una transición que mostró rasgos de continuidad con el régimen militar ${ }^{6}$, el primer gobierno (Patricio Aylwin, 1990-1994) debió esforzarse por consolidar un nivel de gobernabilidad estable y extender la democratización al interior del país. Las reformas del primer gobierno procedieron en primer término a democratizar la administración de los gobiernos regionales. No obstante, para finales de la administración, el gobierno comenzaría a evaluar el estado de la administración pública para proceder a su modernización y el tema sería uno de los puntos clave de la plataforma de gobierno de Frei. En términos de coyuntura social y económica, las diferencias con los casos anteriores son notables. No solo no existía crisis y el país presentaba índices de crecimiento ejemplares, sino que Frei se mostraba orgulloso del país que recibía. El Presidente subrayaba que Chile había crecido en los últimos diez años a una tasa promedio superior al $6 \%$ anual. Remarcaba que, en este mismo período, las exportaciones aumentaron a un ritmo superior al $9 \%$ o que la inflación había disminuido desde más del $20 \%$ anual a niveles del orden del 13\% en 1992 y 1993 (Frei 1994). Asimismo, los datos oficiales a la hora de la asunción de Frei indicaban que la tasa de desempleo había disminuido del $17 \%$ de promedio en la década de los ochenta a un índice del 5\%.

Ante estas realidades diversas, los posicionamientos discursivos de los mandatarios, para legitimar la necesidad de reformas, fueron muy distintos. En el caso argentino, la situación del país era propicia para implantar un cambio radical. Menem se posicionó discursivamente como un "Mesías que acudía a salvar la Patria" en medio de la consternación. No es casual que las primeras palabras con las que se dirigió a la Asamblea Legislativa fueran:

Ante la mirada de Dios y ante el testimonio de la historia, yo quiero proclamar: Argentina, levántate y anda (Menem 1989).

\footnotetext{
Entre 1973 y 1980, el régimen militar redujo las empresas públicas de 400 a 45. Asimismo, la plantilla de empleados se redujo un 30\% de manera brusca y radical, pasando de 241.000 en 1976 a 131.0001984 (Waissbluth 2006: 22).

6 Para un estudio crítico de las continuidades entre en régimen militar y la democracia y los pactos de la transición, véase Moulian (1997).
} 
La misma frase sería repetida en varios discursos ${ }^{7}$ (Menem 1991b). Menem se presentó como el líder que venía a reconstruir un país destrozado y a "cambiar la historia en la República Argentina” (Menem 1990b). Y la posibilidad de resurgimiento nacional llegaba, en el discurso menemista, en el medio de la tragedia. Para el Presidente, el país había tocado fondo:

El país está quebrado, devastado, destruido, arrasado (...). Estamos en una auténtica situación de emergencia económica y social. (...) Porque toda la ciudadanía sabe que no miento, si afirmo que estamos viviendo una crisis dolorosa y larga. (...) La más terrible de todas las crisis de las cuales tengamos memoria. (...) A la Argentina la sanamos entre todos los argentinos o la Argentina se muere. Se muere (Menem 1989).

Ante un interlocutor desesperado, la situación era óptima para sedimentar un nuevo paradigma. Como explican Novaro y Palermo, "los apoyos o la tolerancia social a las reformas y a la estrategia menemista provinieron (...) de la necesidad de huir de un presente insoportable y (...) del temor a volver a esa situación extrema" (Novaro y Palermo 1996: 235).

Por su parte, Sanguinetti inició su mandato sin verdaderos focos de crisis que pudieran amenazar la estabilidad del país. El gobierno centró discursivamente las reformas en el contexto de la globalización. Los cambios se argumentaban en la necesidad de reorganizar al país y dotarlo de la competitividad requerida para ser rentable en el marco de una economía global y, principalmente, en el Mercado Común del Sur (MERCOSUR).

El fin del siglo sorprende al mundo con una multiplicidad de cambios que se producen de forma acelerada y revolucionaria. (...) Nadie puede ignorarlos sin riesgo de encontrarse como un explorador perdido en la selva (Foro Batllista 1998: 13).

El reconocimiento de la globalización como punto nodal del discurso sanguinettista, la necesidad de comprender el proceso y acomodarse a sus cambios para no quedar en la deriva, implicaba el reconocimiento de un cambio consumado en las posibilidades ideológicas:

Sabemos que todos los empeños para que la economía de mercado fuera suplantada por otros emprendimientos y por otros sistemas, terminaron (...) en el fracaso (Sanguinetti 1995).

\footnotetext{
Esta frase mesiánica sería recuperada en el futuro para hablar de los logros del gobierno. Por ejemplo: "Ese Argentina levántate y anda de 1989, es hoy una realidad" (Menem 1991b).
} 
Este marco de interpretación de coyuntura brindó al gobierno argumentos sólidos para establecer discursivamente la necesidad de modernizar al Estado y prepararlo para la competencia internacional.

Por su parte, el posicionamiento discursivo de Frei presenta rasgos distintivos respecto a los demás casos analizados. Si bien también se encargó de advertir sobre la necesidad de tolerar los modos en los que se había reinstalado la democracia, lo hizo desde una posición de optimismo que se justificaba en la buena marcha del país.

La legitimidad democrática de hoy surge de la legitimidad de una historia común. (...) Gracias a esta herencia común, ustedes no están hoy ante un Presidente que afirma que con él comienza todo de nuevo en Chile (Frei 1994).

Frei no sólo reconocía que el país que recibía no estaba en crisis, como sucedió en distintas medidas en los demás casos, sino que también tomaba la posta de su compañero de partido y, como se explicó antes, vivaba los éxitos del pasado gobierno. Ante este panorama, la prédica a favor de reformas debía centrarse en una estrategia que no dependiera del pánico para justificar los cambios. Así, la gestión de Frei se enmarco en la proposición de que el país tenía una oportunidad histórica: "la de convertirse en una nación desarrollada en el tiempo de nuestra generación" (Frei 1994).

Por primera vez en muchas décadas, nuestro país tiene una estrategia para alcanzar el desarrollo (Frei 1998).

Si el rumbo político, económico y social era el correcto, según los índices, lo que le faltaba a Chile para consolidar su desarrollo era, según la idea esbozada en el discurso oficial, mantener el mismo esquema del gobierno anterior, sumando un nuevo eje a la agenda: la modernización de las infraestructuras y los modos de gestión. Poniendo énfasis en esta idea, se impulsaron discursivamente las nuevas privatizaciones y la reforma de la Gestión Pública, que venía siendo una proposición de la Concertación tanto desde los últimos meses del gobierno de Aylwin como desde la plataforma electoral de Frei.

Pero, más allá de las diferencias, existió una similitud en torno a todos los discursos: la presencia hegemónica del factor económico como legitimador en sí mismo. Si en Argentina lo económico giró en torno al déficit fiscal y la inflación, en Chile se centró en el desarrollo y el crecimiento como motor del país y en Uruguay en la necesidad de mejorar la competitividad para ser productivos y poder instalarse con buen pie en el nuevo orden globalizado. 
Imaginario Político y Discursos Técnicos en las Reformas Administrativas

\section{CONSTRUCCIÓN DISCURSIVA DEL ALCANCE DE LA DEMOCRACIAY EL ESTADO}

Es importante comprender que el discurso que ha caracterizado a los distintos paradigmas de reforma de la administración pública no significa por sí mismo, por lo que el estudio de las ideas en torno a las cuales giran los discursos de implementación de los conceptos técnicos es fundamental para comprender la totalidad del proceso. Conceptos como eficacia, eficiencia, productividad no significan por sí solos, sino que deben estar cargados de sentido a partir de un sistema de ideas al que son incorporados. Es decir, los conceptos técnicos se materializan a partir de un discurso ideológico ${ }^{8}$. Así, la eficacia o la eficiencia, por ejemplo, se implementarán en una estructura organizacional a la que una noción particular de Estado o democracia carga de sentido. Desde aquí, un gobierno construirá las instituciones que considere adecuadas?. Un país podrá suponer que lo óptimo es contar con 7 ministerios, y otro con 15, y dentro de este marco un Estado podrá optar por constituir 5 dependencias dedicadas a aspectos económicos y otro podrá optar por dos o tres dependencias con rango ministerial encargadas de atender aspectos sociales como la pobreza, el acceso a la vivienda o el empleo. Estas decisiones dependerán fundamentalmente del imaginario político de cada caso. Por esto, para analizar las reformas de la administración, y su efectividad, es menester partir del marco de ideas en el cual operan. En este sentido, los discursos oficiales, en los casos analizados, muestran distintas maneras de comprender al Estado y a la democracia.

La historia política argentina del siglo XX estuvo marcada por golpes militares, que se sucedieron desde 1930 hasta la reinstauración definitiva de la democracia de 1983. La vida política "democrática" en este período estuvo fuertemente marcada por el peronismo y la imagen personalista del General Perón. Tras la dictadura militar, volverían a imponerse regímenes de marcado rasgo personalista como una cuestión natural. Al mismo tiempo, tanto la sociedad como la clase política se decantarían por una idea de democracia absolutamente delegativa (O’Donnell 1994) en la que el líder recibiría carta blanca para actuar. Justamente, uno de los puntos más controvertidos de la reforma menemista fue la manera en la que el Presidente impuso sus decisiones tras la aprobación de las leyes de Emergencia Administrativa (Ley 23.696) y Emergencia Económica (Ley 23.697), con las cuales logró, por consenso político, medios legales para impulsar leyes sobrepasando el control del Legislativo. La fragilidad institucional y el contexto financiero no sólo justificaron la imposición de un discurso articulado en torno a variables eco-

\footnotetext{
8 Se puede estipular en principio que, por ejemplo, todo proceso humano está signado por la eficacia, aunque más no sea en el ser eficaz para ser ineficaz. La medición o evaluación de la eficacia necesariamente dependerá de un sistema de ideas particular y arbitrario.

9 En este aspecto ingresa desde el tipo de gobierno hasta su estructura. La elección de un sistema legislativo unicameral o bicameral dependerá de un imaginario político sedimentado.
} 
nómicas, sino que fueron el argumento utilizado para lograr consenso político sobre un nuevo modo de accionar institucional y dirigencial centrado en la omnipotencia del Poder Ejecutivo. Concretamente, las medidas fueron establecidas por decretos de necesidad y urgencia ${ }^{10}$ sin interferencia del Poder Legislativo. Por esto, muchos autores cuestionan la "legitimidad de la reforma"11 por el actuar "decisionista" del Presidente (Ghio y Etchemendy 1998: 42-49) o por el sentido "excluyente" y "unilateral" de los cambios (Repetto 2001: 14).

La manera en que Menem defendió su actitud fue alegar que la urgencia de resultados requería medidas veloces que impedían obtener "un pronunciamiento legislativo expeditivo en tiempo". Si bien son válidas las críticas al modo de ejercer el poder menemista, es necesario destacar el consenso político tácito que existía al respecto. También sirve a modo de alegato el hecho de que la posibilidad de sobrepasar la instancia legislativa y crear decretos con fuerza de ley fue implementada, más tarde, en la Reforma Constitucional de 1994. No obstante, es verdad que el presidente se mostraba dispuesto a evitar las funciones del Legislativo si este se oponía a los planes de Ejecutivo. Durante un acto, en noviembre de 1991, Menem se refería a las críticas recibidas por su estrategia de gobernar por decreto, incluso cuando los legisladores se negaban a aprobar los planes del gobierno:

(...) Lo importante es que ese decreto tiene vigencia y cuando nuestros amigos del Parlamento quieran tratarlo, que lo traten, pero mientras tanto tiene plena vigencia y de este camino nadie nos va a desviar (Menem 1991b).

La estrategia discursiva para justificar la intromisión entre poderes era igualar los planes de gobierno con el deseo de la mayoría de ciudadanos:

Nos han tratado de totalitarios. ¡Si es que el 86 por ciento está avalando este decreto! Prefiero ser totalitario de esta forma y no democrático con un 20 o un 10 por ciento de la población (Menem 1991b).

Pero por otro lado, el caso argentino presenta una particularidad que lo diferencia de los otros dos casos analizados: el grado de continuidad entre el discurso electoral y el discurso presidencial es nulo. Una vez en el poder, Menem rompió

\footnotetext{
10 Los decretos de necesidad y urgencia en Argentina son aprobados directamente por el Presidente y pueden tener fuerza de ley.

11 Los decretos de necesidad y urgencia emitidos por el Presidente en Argentina tienen los efectos jurídicos de una ley. No obstante, hasta la reforma de 1994, la Constitución Nacional no atribuía al Poder Ejecutivo la facultad de emitirlos. Por lo tanto, su constitucionalidad era efectivamente dudosa. A pesar de esto, la utilización de esta figura existía y estaba reconocida tácitamente. No obstante, lo significativo del gobierno de Menem fue la magnitud de este accionar: Alfonsín emitió 8 decretos de necesidad y urgencia durante su mandato, mientras que Menem superó las dos centenas.
} 
abruptamente con su discurso de campaña, signado por una enunciación populista e ideas nacionalistas ${ }^{12}$, y se abocó a defender la economía de mercado. En su discurso frente a la Asamblea Legislativa, en julio de 1989, Menem ya anunciaba medidas económicas:

Esta economía de emergencia va a vivir una primera instancia de ajuste. De ajuste duro. De ajuste costoso. De ajuste severo (Menem 1989).

Para salir de la crisis, Menem advertía, además, que se dependería de la inversión foránea:

Vamos a ser generosos y amplios al convocar al capital extranjero (Menem 1989).

En 1990, el rumbo ideológico del discurso menemista era claro. Las nuevas ideas en torno al Estado y sus funciones tuvieron un acentuado tinte neoliberal. Aunque las medidas reformulaban los alcances institucionales del país, fueron implementadas sin consulta popular ni discusión legislativa. En pos de un Estado mínimo, las anteriores nociones estatales se consideraron "viejas y obsoletas", obstáculo para el desarrollo del país:

El Estado no ha fracasado. Ha fracasado el estatismo. (...) El Estado como organización política y categoría histórica, sigue teniendo validez. Las que ya no tienen vigencia son sus actividades como empresario, como productor, como industrial y como comerciante (Menem 1990a).

Por ello se implementaría:

Un Estado dedicado pura y exclusivamente a sus funciones específicas (...). Ni petróleo, ni gas, ni teléfono, ni carreteras, ni usinas, ni aerolíneas, ni televisión (...) Nunca más el Estado incursionando o interviniendo en áreas que no le competen (Menem 1990b).

Las nuevas funciones específicas del Estado eran "regular los marcos generales de convivencia, generar las condiciones materiales para la producción, inducir la inversión, orientar el crecimiento y fiscalizar la distribución" (Menem 1992). Asimismo, a la hora de reestructurar la organización institucional, el gobierno consideró que las funciones primordiales se resumían a la recaudación de tributos. Por ello, en el programa de Reforma, si bien existe la queja sobre el incremento de la

12 Para ampliar sobre el discurso menemista en general, véase Sigal y Verón (1988). 
planta de empleados en general, se cuestiona el poco crecimiento de la plantilla de los organismos responsables de la recaudación, que contaban con "apenas 22.000 agentes, menos del 4\% del total de la APN" (Domeniconi, Gaudio, y Guibert 1992: 11). Según el gobierno, "este grupo de organismos descentralizados es de los de mayor relevancia para el funcionamiento de las políticas públicas, no dispone de dotaciones de magnitud acorde con la importancia de sus funciones" (Domeniconi 1992: 15) ${ }^{13}$.

Por otra parte, Menem sedimentó una idea de política en la que los costes sociales no se planteaban como un problema, sino como una necesidad de cumplir objetivos primordiales. Pensar en despidos para descongestionar la administración pública o implementar recortes salariales y recortes de presupuestos en educación y salud fueron cuestiones que se incorporaban dentro de lo hacible.

La democracia en Uruguay se entendió de manera muy distinta. Tras la reinstauración de la democracia, en 1985, el punto nodal del discurso de Sanguinetti fue el objetivo primordial de consolidar el nuevo sistema. La propuesta se anclaba en el pasado y en la cultura nacional y giraba en torno a un núcleo ideológico central que equiparaba la identidad nacional al sistema de gobierno.

Esta República que nació para la democracia (...), la gran causa (nacional) que nos convoca desde el día en que nació este país. (...) Ortega decía: Hay verdades del destino y hay verdades teóricas. Las verdades teóricas nacen de la discusión, nacen de la razón, se nutren de ella, viven de la discusión, se vigorizan con la discusión. Hay otras verdades que son verdades de destino, esas no se discuten; se asumen porque esa es la identidad propia, eso se es o no se es y eso está antes de lo que se discute. Y para nosotros los uruguayos la democracia es una verdad de destino, es un destino irrenunciable (Sanguinetti 1985).

Además de ser la característica del ser nacional, la democracia era presentada en el discurso sanguinettista como el estandarte por el cual Uruguay era reconocido en el mundo entero.

La identidad nacional para nosotros fue (...) un valor político y cultural. Los uruguayos fuimos eso, una expresión de democracia dentro del Río de la Plata. Esa también es nuestra definición internacional. (...) Esta es la identidad del Uruguay. Nuestro país es eso o no es nada (Sanguinetti 1985).

\footnotetext{
13 En consecuencia, la reducción de personal no fue discriminada, sino que se hizo una importante excepción con la Dirección General Impositiva y la Dirección de Aduanas. El número de empleados de estas dos agencias se incrementó de 14.000 a 25.000 (Domeniconi 1996: 13).
} 
En este sentido, Panizza destaca el "carácter fuertemente restaurador" del regreso de la vuelta de la democracia (2002: 76). Todas las instituciones congeladas durante el gobierno militar fueron reincorporadas y las impuestas por la dictadura desmanteladas. La Constitución de 1966 se readoptó sin cambios y la primera elección arrojó resultados muy similares a la última elección democrática previa al golpe, por lo que se puede aseverar que "los años de dictadura militar no debilitaron, sino por el contrario reforzaron, a la democracia como uno de los significantes clave del imaginario político uruguayo" (Panizza 2002: 77). La idea de democracia como identidad nacional sería recuperada como valor hegemónico tanto por Lacalle como por Sanguinetti en su segundo mandato.

Justamente este carácter democrático se vio en el plebiscito de 1993, en el que el pueblo se opuso al proyecto privatizador impulsado por la gestión de Lacalle, que aceptó la consulta popular para ver la legitimidad de sus planes. El resultado modificó sustancialmente tanto la agenda del gobierno como las posibilidades discursivas. De esta manera, aunque el gobierno de Sanguinetti también abogaba por privatizar y reducir el Estado, enunciaba las propuestas desde la necesidad de dotar al país de una estructura acorde a la nueva época. Así, el Estado podría seguir, con éxito, ocupando el centro de la estructura social, como históricamente aclamó el batllismo.

Al mismo tiempo se discute la amplitud del papel del "Estado Benefactor", que marca el fin de un proceso que puso a cargo del Estado los riesgos sociales. Se le exige ahora la reducción de su costo operativo, la disminución de su ámbito regulador y el abandono de la mayor parte de sus actividades comerciales e industriales. El debate de ideas se hace apasionado y apasionante entre quienes sostienen la tesis del Estado mínimo y quienes creemos que el Estado, aún cambiando los roles, no puede abandonar su condición de garante de los equilibrios de la sociedad (Foro Batllista 1994: 14).

La misma estrategia se utilizó para distanciarse de la tradición discursiva que subrayaba la eficacia del sector privado por sobre el estatal. En este sentido, el gobierno advertía que el avance privado se legitimaba ante la ineficacia del gobierno para atender las necesidades de la ciudadanía y, para evitarlo, las modificaciones estipuladas se tornaban imprescindibles.

Hay un esfuerzo que la sociedad debe hacer y (...) no puede permitirse ninguna debilidad, porque cuando el Estado deja de ser eficaz aparece la reacción privada, y eso es lo que no ha ocurrido en este país. Y no ocurrirá (Sanguinetti 1995). 
En definitiva, el imaginario político uruguayo continuó el discurso histórico del batllismo tradicional que colocaba al Estado como motor político y núcleo de la organización institucional, social y económica (Panizza 2002). Por otra parte, vale destacar que la coherencia en términos de continuidad entre el discurso de campaña y el discurso presidencial fue total, lo que marca una diferencia importante en términos de imaginario político con el caso argentino.

En lo referente a Chile, el respeto al discurso de la plataforma electoral fue muy alto, tanto en el primer gobierno de la Concertación, como en el siguiente de Frei. Asimismo, entre el discurso de Aylwin y el de Frei existe una narratividad continuada y una concordancia plena en los alcances del Estado y la idea de democracia. El discurso de Frei retomaba la concepción de Estado defendida en la plataforma electoral. Esto es, un Estado agente del Progreso, como también había estipulado la gestión anterior.

Es evidente que los problemas del país no podrán resolverse exclusivamente por mano del Estado; pero éste juega un papel fundamental en la sociedad moderna (Concertación de Partidos por la Democracia 1993).

El rol del Estado era "generar el clima propicio para que el sector privado pueda desenvolver sus actividades" (Frei 1998). El Estado se debía encargar de regular los mercados, buscar nuevos mercados para los productos chilenos y apoyar los esfuerzos privados que buscaran mejorar la capacidad de los pobres para ganarse la vida por sí mismo. No obstante, tal como pasó en Uruguay, donde el imaginario político se sustentaba en las ideas del batllismo clásico, en Chile la Concertación tampoco aceptó la concepción neoliberal del Estado mínimo ${ }^{14}$, aunque la doctrina económica se centraba en las ideas del libre mercado y la apertura económica.

No comparto la visión simplista según la cual modernizar el Estado supone despojarlo de todo poder y autoridad, reduciéndolo prácticamente a la nada. Queremos un Estado moderno, pero también fuerte, democrático y eficiente en su gestión; que pueda relacionarse fluidamente con el sector privado, pero a partir de reglas claras sobre las incompatibilidades entre el servicio público y el mundo de los negocios privados; que pueda aspirar a tener los mejores profesionales y técnicos del país, y que en el ejercicio de la función pública sus talentos y capacidades creadora estén al servicio real de la gente (Frei 1994).

\footnotetext{
14 Es de recordar en este punto que, en el imaginario político y social chileno, hablar de neoliberalismo automáticamente se relaciona con el gobierno dictatorial, que implementó sus reformas amparado en esta ideología. Tras el advenimiento de la democracia, a pesar de las continuidades, lo neoliberal y el neoliberalismo fue excluido de las posibilidades discursivas hegemónicas.
} 
El ejemplo chileno se suma al uruguayo para mostrar que existió una amplitud de ideas diversas a la hora de sedimentar las ideas sobre la política. Las libertades en el sentido de decidir el destino de cada orden político se ven, sobretodo, en las distintas nociones de democracia construidas, y defendidas, por los gobiernos analizados. Las actividades y funciones que debía cumplir el Estado, entonces, son connotadas, discursiva y programáticamente, por los valores que cada mandatario une a la democracia como forma de gobierno. En el caso de Frei, el gobierno chileno continuó la línea iniciada por Aylwin de entender a la democracia como un orden social que sólo podía perpetuarse en el tiempo con altas dosis de justicia social.

No existe democracia estable y posible en el largo plazo, con desigualdades extremas en la distribución del ingreso y de las oportunidades sociales. No existe democracia posible con porcentajes significativos de la población viviendo en la extrema miseria (Frei 1996).

Era desde el Estado donde este discurso debía materializarse. A consecuencia de este marco de interpretación de coyuntura, la pobreza se convirtió en un punto nodal en el discurso oficial, que volvió a articular la democracia en torno a valores de equidad y justicia social, advirtiendo sobre las ventajas económicas y productivas que la lucha contra la pobreza generaba para los demás sectores sociales.

Si no actuamos con decisión en estos planos, seremos responsables de estar perpetuando la existencia de dos países: un Chile moderno, rico y próspero, y otro añejo, atrasado y pobre. Superar la pobreza es algo más que proporcionar un poco de bienestar a las personas. Es una obligación solidaria de todos los chilenos que hemos podido crecer y desarrollarnos con aquellos compatriotas que no han tenido oportunidades o que no han sido capaces de aprovecharlas. Nuestra generación de políticos será la responsable de responder ante la historia por lo que seamos capaces de hacer para que Chile sea una sola patria (Frei 1994).

Sabemos que sin crecimiento no puede existir progreso material; pero sin una activa política social sería imposible lograr el bien común y sostener el crecimiento. (...) No basta con que el país avance; cada familia tiene que hacerlo (Frei 1998).

Como se ve, algo que a menudo se ha interpretado como resultado de una buena administración, como es la extrema reducción de los índices de pobreza en Chile desde la reinstauración de la democracia, no debe ser analizado simplemente desde cuestiones técnicas, sino desde el imaginario social y político hegemónico sedimentado por la clase política. Es comprensible, bajo este esquema de 
prioridades discursivas, que las políticas de lucha contra la pobreza hayan logrado resultados óptimos cuando se trataba de un valor elemental dentro del programa. Las ideas rectoras de los programas de gobierno determinan, en el caso de la administración, no sólo los principios rectores de la función pública y de cómo llevarla a cabo, sino también las instituciones que conformarán la estructura organizacional del Estado. Que un gobierno decida impulsar una institución centrada en la lucha contra la pobreza, o que decida fortalecer los organismos encargados de recaudar impuestos, como sucedió en Argentina, es una decisión amparada en las ideas rectoras que determinan el marco de interpretación de coyuntura. En este sentido, del mismo modo que se pueden estipular concepciones sobre el Estado, su naturaleza y su rol, o sobre la democracia, que luego determinar las formas de administrar una gestión pública, también los parámetros determinados por la cultura política, y por la forma de entender la democracia, normativizan lo hacible y tolerable en el marco de una gestión de gobierno. Una característica importante que diferencia a los casos chileno y uruguayo del argentino, es el respeto discursivo de los presidentes a los programas electorales de sus respectivos partidos y la precaución de no impulsar medidas que determinen costos sociales graves.

El análisis del caso chileno muestra la importancia de analizar las ideas rectoras del imaginario político desde una visión amplia y compleja. El combate contra la pobreza fue presentado como la posibilidad de consolidar la economía y mantener los índices de crecimiento. Si bien es de destacar el apoyo de los sectores más privilegiados que el gobierno consiguió para impulsar programas sociales de reducción de la pobreza -incluyendo el aumento del gasto social y la reforma impositiva-, es necesario interpretar esta situación no sólo desde los índices satisfactorios de reducción de la pobreza, sino también desde la amplia brecha entre los ingresos de los más pobres y los más ricos. A pesar de disminuir la pobreza, Chile se ha mantenido en el período analizado como uno de los países con peor distribución de la riqueza de Latinoamérica y su coeficiente de Gini se ha mantenido estable ${ }^{15}$. Es decir, en medio de una coyuntura de crecimiento económico constante, la disminución de la pobreza fue acompañada por un aumento proporcional de los ingresos de los más ricos. Este dato no es menor para comprender el apoyo de todos los sectores a los planes sociales del gobierno.

Por otro lado, era lógico que los más beneficiados discursivamente, tras una transición en la que los militares continuaron dirigiendo importantes nichos de

15 El coeficiente de Chile apenas se ha movido desde la llegada de la democracia. En 2007, Chile tenía un índice de 53,8, lo que lo convertía en el 17mo país más desigual del mundo. En definitiva, a medida que la pobreza se fue reduciendo en Chile tras la reinstauración de la democracia, la riqueza de los más pudientes aumentó de manera proporcional. La progresión del índice en el período analizado fue de 0,554 (en 1990), 0,553 (1996), 0,559 (2000). Como se ve, entre 1990 y 2000 incluso subió. 
poder, fueran quienes habían resultado más perjudicados por el pinochetismo. De alguna manera, el continuar la misma línea de la dictadura en términos de política económica implicaba legitimar la forma drástica en la que el neoliberalismo fue implantado en el país. Sin este discurso integrador, y sin acciones efectivas en este sentido, difícilmente la nueva clase política chilena hubiese conseguido consolidad su posición y su legitimidad.

\section{LEGITIMACIÓN DE LA REFORMA Y ROL DE LA ADMINISTRACIÓN PÚBLICA}

Menem fue quien más claramente definió a la administración como la base del buen gobierno y el progreso:

El problema argentino no es (...) por falta de desarrollo, sino un problema que radica en la falta de administración (Menem 1991a).

Yo vengo sosteniendo desde hace mucho tiempo (...) que Argentina no es un país subdesarrollado, sino que se trata de un Estado totalmente subadministrado (...). A partir de esto hemos puesto en marcha este proceso de transformación del Estado, que va a darle la posibilidad a Argentina de organizarse (Menem 1990c).

En este marco surgen diferencias importantes en torno a la cultura política que determinaron las maneras de impulsar los cambios, y sus consecuencias. En Chile, al igual que en el caso uruguayo, las ideas sedimentadas en el imaginario político no incorporaban dentro de lo hacible la posibilidad de despidos masivos y recortes abruptos de personal, como sucedió en Argentina. Pero si bien el discurso oficial de la Concertación incorporaba imperativos morales que colocaban al Estado como el garante de la calidad de vida de los ciudadanos y defensor de los más débiles, esto se estipulaba desde un marco general en el que lo económico -o el desarrollo económico- figuraba como principio motivacional y valor finalista último. En Uruguay, el Estado se reconocía garante de la calidad de vida de los ciudadanos, por lo que preservar la buena convivencia entre los habitantes era una exigencia. En Argentina se naturalizó utilizar como variable de ajuste el sueldo de los empleados públicos y los presupuestos de sectores básicos como la salud o la educación. Las medidas eran acompañadas con un discurso pro-patriótico que alentaba a hacer esfuerzos para reacomodar las finanzas. Por el contrario, en Chile, reequilibrar el valor real de los sueldos, que se había dilapidado durante la dictadura, fue un imperativo desde la reinstauración de la democracia.

En cuanto a los diagnósticos sobre el estado de la administración, todos los discursos coincidieron en que los esquemas antiguos eran lentos y poco funcio- 
nales para hacer frente a las necesidades de la población. Respecto a la administración central, Argentina sumó al diagnóstico una crítica al número de dependencias y a la plantilla de personal, a la que consideró excesiva. Uruguay también consideraba que, para reacomodar la plantilla a un nivel óptimo, era necesario suspender los ingresos a la carrera administrativa. En todos los casos, la administración recibida era considerada un obstáculo para responder a los problemas de la coyuntura y propiciar el desarrollo. Asimismo, a partir de las concepciones de Estado defendidas por cada administración, se estipularon tareas que correspondían al Estado y tareas que debía ser tercerizadas o descentralizadas. De esta manera se incorporaba un nuevo tipo de propiedad: lo público no estatal (Bresser 1996, Cunill 1997, CLAD 1998). Ante esta situación, el discurso en los tres casos fue similar: responder de mejor manera a la necesidad de los ciudadanos, ser eficientes, eficaces, productivos y resolutivos.

En este marco, se procedía a sedimentar una racionalidad-técnica enfocada a resultados en detrimento de la anterior racionalidad-legal a partir de evaluación por normas procedimentales. El nuevo marco de evaluación implicaba la necesidad de recurrir a indicadores medibles para proceder a la evaluación constante del desarrollo. Por supuesto, las ideas cumplieron un rol fundamental en este ámbito. No sólo porque la estructura técnica no define por si misma qué es necesidad de los ciudadanos ni cuáles son los indicadores a tener en cuenta a la hora de proceder a las mediciones. Además, el imaginario político de cada país determinó las formas en las que se desarrollaron tanto las privatizaciones como la tercerización de actividades. Los procesos licitatorios y las ventas, así como el marco jurídico en el cual desarrollarían sus actividades, también necesariamente estuvieron signados por lo hacible y tolerable dentro de cada ámbito.

Por ejemplo, las privatizaciones en Argentina y en Chile estuvieron marcadas por discursos completamente distintos. Mientras en Argentina lo importante era la velocidad para adquirir divisas y equilibrar el déficit fiscal, las privatizaciones impulsadas por el gobierno de Frei se dieron bajo un discurso de modernización de la gestión y mejora de la rentabilidad y la producción. Las privatizaciones se justificaban y presentaban como manera de cumplir metas empresariales que, dependiendo de capital público, difícilmente podrían realizarse. La privatización era, entonces, un instrumento para cumplir los objetivos de infraestructuras necesarios para impulsar ese desarrollo y concretar la oportunidad histórica de la que Frei habló desde su asunción. Esta idea determinó que no se incluyeran dentro de lo privatizable empresas que se consideraban podrían ser redituables desde una buena gestión pública, como CODELCO ${ }^{16}$. En Uruguay, mientras tanto, el Estado mantuvo las empresas de servicio a su cargo. No obstante, se fue permitiendo el ingreso del capital privado al momento de incorporar nuevos servicios, como la puesta en funcionamiento de la telefonía móvil.

16 Para un análisis de la influencia de esta misma idea durante la apertura de mercado y el impulso de las privatizaciones en el régimen militar véase Cypher (2004). 
Por otro lado, existe una concordancia en los discursos oficiales en el modo de aprehender la realidad diagnosticada. Las descripciones de las situaciones sobre la que se desea actuar se resumen estrictamente a lo que existe jurídicamente y se estipulan en medio de un contexto de causa-consecuencia unidireccional donde no interviene, por ejemplo, la posibilidad de dislocaciones a la hora de poner en práctica los nuevos discursos. Así, se parte de una idea de interpretación racionalobjetiva de los nuevos discursos y se niega la posibilidad de resignificaciones por parte de los actores intervinientes. Esto se relaciona con la construcción del sujeto planteada en los distintos programas; esto es, un ser humano hiper-racional objetivo (cuya motivación se da exclusivamente en procesos de racionalidad instrumental). Esta simplificación trastorna la continuidad narrativa, tanto en la teoría como en la práctica.

\section{LOS OBSTÁCULOS NARRATIVOS DE LOS DISCURSOS OFICIALES EN LOS CASOS ANALIZADOS}

En definitiva, la gran laguna de los discursos oficiales es centrarse en una visión racional legal de las estructuras organizacionales que deja fuera del análisis a las instituciones informales (North 1990) ${ }^{17}$. El diagnóstico sólo se realiza sobre las normas escritas, entendiendo lo jurídico como lo existente. Siguiendo a Scott (1990), se trabaja siempre sobre el texto público ${ }^{18}$ y no intervienen en el debate las ideas que actúan sobre el texto oculto.

Sin embargo, los discursos académicos han apuntado a reconocer la informalidad y el poco apego a las leyes en el interior de la función pública latinoamericana. Allen Schick (1998) alertó sobre el carácter informal y clientelista del liderazgo político y la ausencia de una mentalidad de respeto espontáneo a los contratos (Schick 1998: 127). Por su parte, Prats (1999a) hace alusión al sistema de patronazgo (spoil system) que se aprecia en las administraciones públicas de la región, donde se privilegia la confianza y el favoritismo político a los méritos profesionales. Mientras tanto, Ferraro recuerda que las irregularidades no se producen por falta de normas claras al respecto, sino por 'modos de hacer' culturales (Ferraro 2007: 116). Como explica Oscar Oszlak,

17 Para North, las instituciones forman la estructura de incentivos de una sociedad, son las reglas de juego. Las instituciones pueden ser formales o informales. Las reglas formales son sólo una parte de la matriz institucional que debe complementarse con restricciones informales. Rutinas, costumbres y tradiciones son palabras que usamos para referirnos a la persistencia de reglas informales.

18 Scott utiliza el término "en escena" para referirse a aquellos lugares en los que prevalecen las condiciones hegemónicas. Mientras "tras las bambalinas" se desarrollan ocultamente los intereses de grupo, sobre la "escena" los grupos dominantes desarrollan aquellas acciones tendientes a legitimar el orden social y buscar de consenso social necesario para perpetuar su hegemonía. En este sentido, "el texto público es (...) el autorretrato de las elites dominantes tal como ellas quieren ser vistas públicamente" (1990: 18). De esta manera, mientras el texto público está marcado por la estrategia de marketing, el "texto oculto" con los intereses reales de cada sector. 
...cada cultura tiene su propia visión sobre cómo deben comportarse los funcionarios públicos y determina la legitimidad de sus roles. El nepotismo, la venalidad, el ausentismo -entre otras prácticas que Parsons denomina particularistas- son propias de ciertas culturas o, en todo caso, se hallan más difundidas en unas que en otras (Oszlak 1999: 19).

Y en Latinoamérica, algunas de estas prácticas de mantienen dentro de lo hacible y tolerable por el imaginario político.

Las advertencias sobre la necesidad de ser cautelosos a la hora de abusar de las soluciones técnicas, y suponer que por sí solas tienen potencial suficiente para modificar actitudes culturales, se justifican en los tremendos errores e inconvenientes que dejaron antiguos procesos similares. Como explica Prats,

... la reforma administrativa de los 60 y los 70 , en la que tanto invirtió América Latina, ha dejado préstamos aún no pagados, escuelas y facultades de administración, organismos administrativos de reforma y hasta organizaciones intergubernamentales sobre el tema; pero no consiguió apenas despatrimonializar nuestras administraciones ni mejorar su eficacia y eficiencia, su transparencia, su previsibilidad, su sometimiento a la ley, el acceso a las mismas de los ciudadanos, y la responsabilidad de sus autoridades y funcionarios. Cuando llegó la gran crisis de los años 80 resultó que las jóvenes administraciones públicas latinoamericanas tan trabajosamente construidas a lo largo de las dos décadas anteriores, en lugar de ser un recurso del desarrollo, habían pasado a formar parte de la agenda de problemas" (Prats 1999b: 119).

Lamentablemente, la historia pareciera repetirse, por lo que se torna urgente rever la narratividad histórica y la coherencia discursiva de este campo político y académico, reconociendo de antemano que, ante una sucesión de fracasos de este tipo, una revisión epistemológica puede ser lo más adecuado.

Es necesario analizar el estado de la administración desde las ideas que determinan la sedimentación de instituciones informales en el seno de cada dependencia. Asimismo, reconocer la existencia de instituciones informales es entender que no se pueden estipular programas universales que puedan dar cuenta de todos los conflictos culturales particulares de cada caso. En este sentido, el discurso analítico de las reformas obvió incluir dentro de su esquema de análisis el rol de las ideas, lo que determinó que se actuara sobre concepciones del poder y lo político (y su desarrollo) limitadas. Para analizar esta situación se debe recordar que, en un principio, los proyectos han apuntado a mejorar el rendimiento de 
administraciones desmotivadas, informales, corruptas, clientelares o patrimoniales. No obstante, se apuntó a buscar soluciones desde aspectos eminentemente institucionalistas y no se partió de una comprensión global del alcance del poder. Es necesario reconocer al poder en toda su dimensión, y la vida política desde la complejidad de sus decisiones y necesidades. Si, por ejemplo, se comparan las coincidencias entre el programa de reformas menemista con la literatura académica, o más precisamente con el programa propuesto por el CLAD ocho años después, se ve perfectamente que los políticos saben perfectamente qué se debería hacer. Pero no se hace lo necesario. No obstante, no hay que diabolizar a los políticos por esta situación, sino comprender que la competencia política implica mucho más que hacer lo que sería óptimo para el desarrollo de las políticas públicas (Geddes 1994). Es así como, en medio de todas las condiciones que pudieran hacer falta para impulsar una reforma, una es fundamental y excluyente: la necesidad de voluntad política.

En los casos analizados, la voluntad se dio ante la necesidad de buscar canales de legitimación de la propia clase política $-\mathrm{y}$ de cada gobierno en particularcomo clase dirigente natural, y contrarrestar los focos potenciales de desprestigio. Habermas advierte que todo orden político intenta, y debe, dotarse de legitimidad (1973, 1976), fundamentando retóricamente su régimen de dominación o de ejercicio del poder. De esta manera, la política puede entenderse como una retórica del poder; y retórica se refiere a las diferentes estrategias discursivas que producen poder (Sexe 2001). Si en Argentina Menem debió equilibrar a cualquier coste la economía nacional para que la sociedad comprendiera que no era una quimera vivir en democracia con posibilidad de ahorro y sin hiperinflación, y en Uruguay los partidos tradicionales (Colorado y Blanco) debieron aceptar modificar algunos de sus vicios políticos y modernizar el Estado ante el surgimiento de una nueva fuerza que rompió el esquema bipartidista histórico ${ }^{19}$; en Chile la clase política democrática debió justificar la transición pactada y brindar mejoras palpables a aquellos sectores que, habiendo sido víctimas de la dictadura, veían como el pinochetismo mantenía lugares de poder 'democráticos' y conseguía legitimar el modelo impuesto por la fuerza ${ }^{20}$.

Si bien, desde lo discursivo, se alegaron necesidades a favor de impulsar cambios, la situación fue más un cambio de maquillaje que una reforma de fondo. Es

\footnotetext{
19 La década del noventa se caracterizó por la consolidación del Frente Amplio como nueva fuerza política de nivel nacional. En las elecciones de 1994, el Frente Amplio (30,6\% de los votos) prácticamente empató a las fuerzas tradicionales: el Partido Colorado (32,3\%) y el Partido Nacional $(31,2 \%)$.

20 A juzgar por los resultados electorales, todas las acciones de reformas y los caminos elegidos se enmarcaron dentro de lo tolerado por la cultura política de cada país. En las elecciones siguientes a los procesos de reforma, los tres gobiernos lograron la continuidad política. Frei fue sucedido por Ricardo Lagos, también de la Concertación; Sanguinetti por el también colorado Jorge Batlle, y Carlos Menem logró la reelección.
} 
de destacar entonces que un argumento académico en torno al por qué impulsar una reforma de al administración y eliminar los vicios culturales políticos tradicionales debería responder a la pregunta de cuál es el beneficio que pudiera lograr la clase política limitando sus prácticas culturales para el ejercicio del poder. No ha existido, a lo largo de los veinte años de reforma una justificación convincente que justificara la necesidad de eliminar el clientelismo no desde una supuesta posición ético-moral sino desde una comprensión global de la eficacia de las herramientas de poder que se desean eliminar. Así, el discurso oficial, actuando sobre el texto público, no incluyó dentro de sus análisis las ideas sobre las que se asienta el texto oculto y mantuvieron la misma lógica clientelar. Muy por el contrario, el discurso negó la realidad y estipuló argumentos que la encubrieran.

El caso del clientelismo en Chile muestra un claro ejemplo de esta situación ${ }^{21}$. La necesidad de impulsar reformas allí cobró fuerza tras las denuncias de corrupción a finales del gobierno de Patricio Aylwin. Entre 1993 y 1994, la Contraloría General de la República investigó un total de 241 denuncias por corrupción en la administración pública (Rehren 2000: 158). Ante la situación, el gobierno no dudó en defender la integridad de los funcionarios públicos y, en distintas oportunidades, arremetió contra las malas intenciones del periodismo que, según opinaba, aprovechaba las situaciones para tejer hipótesis conspirativas y manchar el honor de los funcionarios antes de que la Justicia dictaminara la veracidad o no de la denuncias.

Tengo la convicción de que la Administración Pública chilena es honesta y proba. Podrá tildársela de lenta, engorrosa, de tramitadora a veces, y aún, en muchos casos, de ineficiente; pero no de falta de honradez. Sus funcionarios, generalmente mal remunerados, que a menudo deben intervenir en asuntos en que hay comprometidos cuantiosos intereses, observan una honorabilidad, con frecuencia reconocida en el exterior, que debe ser justo motivo de satisfacción nacional (Aylwin 1993).

Chile es una nación que tiene una tradición muy honorable de servicio público, y ese patrimonio moral debemos cuidarlo y protegerlo. Las denuncias que se publican sobre irregularidades y transgresiones, con

\footnotetext{
21 Por cuestiones de espacio, el análisis de este parágrafo pondrá el énfasis en el caso chileno, aunque situaciones comparables se pueden apreciar en los casos argentino y uruguayo. La elección del caso chileno como núcleo del análisis no es arbitraria, sino que se trata de un análisis crítico de la supuesta mejor (o más eficiente) administración pública de la región. Es necesario advertir que no se trata aquí de desprestigiar a la administración chilena ni de negar sus buenos resultados, sino que se parte de mostrar cómo en aquella administración que la literatura coloca en el estrado del ejemplo a seguir también se aprecian niveles de incoherencias narrativas, estrategias de construcción de la realidad limitadas, y un apego total a la racionalidad técnica que hacen del discurso en torno a la reforma de la administración un relato semánticamente precario.
} 
ser graves, no son evidencias que permitan sostener, en modo alguno, que estemos ante una situación generalizada o sistémica de corrupción. Sin embargo, debemos ser prudentes y cuidar nuestra patria con sentido de futuro. No olvidemos que sobre ella rondan el peligro del narcotráfico y de otros negocios ilícitos (Frei 1994).

Justamente, una de las primeras medidas del gobierno de Frei fue la creación de la Comisión Nacional de Ética Pública para reflexionar en torno a cómo promover el fortalecimiento de la probidad, la transparencia y la dignidad del servicio público ${ }^{22}$. Pero en ningún momento se reconocieron fuentes de clientelismo o acciones en contra de la ley en el interior de la administración. En este sentido, cobra especial importancia el trabajo de Agustín Ferarro (2008), quien demuestra el alto grado de clientelismo en el servicio civil chileno. El autor destaca cuatro mecanismos informales de influencia y control del Congreso sobre la burocracia. El primer mecanismo de influencia por parte del Congreso son las recomendaciones que realizan los diputados y los senadores cada vez que se debe cubrir una plaza de la administración pública. Dadas las condiciones en que se designan estos funcionarios, se podría decir que abundan los puestos políticos. Los legisladores sugieren candidatos tanto para la administración central como para las administraciones regionales, ya sea para cargos de alto nivel o nivel medio. Las recomendaciones se realizan directamente a la autoridad jerárquica superior de quien dependerá el empleado designado (Ferraro 2008: 111). En lo que respecta al carácter tradicional del patronazgo legislativo en Chile, distintos autores consideran que fue una característica común en el sistema político ya antes de la dictadura militar (Valenzuela 1977: 152, Agor 1971: 7, 18, Gil 1966: 109). Cabe recordar entonces, que el artículo 57 de la Constitución prohíbe a los miembros del Congreso hacer recomendaciones para cubrir puestos de la administración pública. Aunque el artículo sigue vigente, no se cumple (Ferraro 2008: 112).

El segundo mecanismos de influencia es el desarrollo de redes de contactos entre miembros del Congreso y funcionarios públicos. Al hacer recomendaciones, los legisladores se aseguran poder contar con contactos clave en la administración, de quienes obtener información y recibir consejos de expertos cuando se realicen borradores o debates sobre legislaciones puntuales. Asimismo, cuentan con una herramienta para estar al tanto de todo lo concerniente al interior de cada dependencia. Las recomendaciones para puestos en la administración, por lo tanto, siguen una clara estrategia de clientelismo inteligente. A partir de ellas, los miembros del Congreso consiguen sus redes de contactos, información privilegiada y consejos de expertos para las políticas públicas (Ferraro 2008: 113). Las redes de contactos entre legisladores y funcionarios públicos ya existían antes de la dictadura militar (Agor 1971: 33, Valenzuela 1984, Valenzuela y Wilde 1979).

22 La Comisión contó con la participación de todos los poderes del Estado y representantes de la sociedad civil. Estaba formada por 14 personalidades de la vida política, académica e institucional. 
El tercer mecanismo es la existencia de reuniones frecuentes, formales e informales, entre miembros de los Comités del Congreso especializados en un área de políticas públicas especiales y funcionarios de la administración de las dependencias relacionadas. Los encuentros pueden considerarse reuniones de trabajo en la que ambos bandos intentan sacar provecho para sus trabajos. Así, los ministros tienen reuniones regulares con los miembros oficiales del Comité del Congreso correspondiente con su cartera. A su vez, los miembros de los comités se encuentran periódicamente con los funcionarios públicos de las agencias correspondientes, por lo que existe un feedback continuo y un circuito de información permanente (Ferraro 2008: 114).

Por último, y relacionado con lo anterior, es de destacar la tradición de que los senadores y diputados chilenos se especializan en áreas puntuales, que determina la adquisición de conocimientos técnicos e información clave sobre ese área particular. La especialización incluye el desarrollo de las redes de contactos entre los funcionarios públicos de la esfera de interés (Ferraro 2008: 115).

En definitiva, no se cumplen los concursos estipulados por ley, pero sí existe una negociación cada vez que hay que cubrir una vacante: puede darse el caso de que, siempre por recomendación de senadores, diputados o funcionarios de alto nivel, exista más de un candidato. En estos casos, se realiza una ronda de negociación, y allí ingresan tanto cuestiones meriotocráticas como cuestiones de cuoteo político. Asimismo, en este contexto, difícilmente algún legislador se atreva a recomendar a gente poco capacitada o sin cualificaciones pertinentes por la responsabilidad que implica abrir las puertas de la administración a un contacto político. Del mismo modo, todo recomendado deberá abogar por el prestigio de quien lo recomendó, teniendo por ello que, como responsabilidad ética, demostrar eficiencia y responsabilidad. En este sentido, se puede comprender que, a pesar del alto grado clientelar de la administración chilena, el desempeño de las funciones arroje resultados destacados ${ }^{23}$.

Por último, hay que destacar el rol fundamental que tiene el cuoteo político en el marco de las recomendaciones y el control de la administración. La influencia es controlada rigurosamente por los compańeros de coalición. Si el puesto de Ministro se asigna a un partido, el de Viceministro se asigna a otro. El mismo proceso de cuoteo político se implementa para los niveles superiores de la administración. El cuoteo político ya pertenecía a las prácticas políticas tradicionales antes de la dictadura (Tapia-Videla 1977: 472). El cuoteo político viola el artículo 32, que concede al Presidente de la República el poder exclusivo de elegir los cargos oficiales. Además, la constitución prohíbe al Senado supervisar y controlar al

23 Iacoviello y Zuvanic (2005) destacan, por ejemplo, que Chile, junto a Brasil y Costa Rica, son los países latinoamericanos con mejor índice de meritocracia. 
gobierno (Art. 49). No obstante, es de destacar que el cuoteo es una sistema efectivo para integrar a la clase política entera al sistema de patronazgo y sedimentar las instituciones informales evitando el monopolio del poder en un sector particular del esquema partidario o en una persona. Esta estrategia no se implementa sólo en el Chile: en Uruguay, con el sistema del "3 y 2"24 en los directorios de las empresas estatales, también se recurre al cuoteo para legitimar la informalidad y preservar los canales de patronazgo y clientelismo en el ámbito político. Estas instituciones informales son importantes para conseguir una cohesión profunda en términos de clase dirigente.

La situación tiene varias lecturas importantes. Por un lado, en el imaginario político se reconoce como justo y aceptable actuar de manera contraria a lo que estipula la ley ${ }^{25}$, en este caso para lograr mayor influencia sobre las dependencias públicas. Pero también es importante destacar que los políticos incluso aceptan reconocer esta realidad en el marco de una entrevista científica ${ }^{26}$. No obstante, no se considera dentro de lo hacible la posibilidad de sincerarse públicamente, por ejemplo, con la prensa. Esta situación real muestra que el clientelismo como herramienta de poder puede funcionar de diferentes maneras, y con diferentes resultados a juzgar por el prestigio internacional de la administración pública chilena. En este caso, se trata de un clientelismo selectivo. Por supuesto, en la administración chilena intervienen cuestiones de mérito a la hora de otorgar un puesto, pero sólo se accede a la competencia meritocrática tras sortear el obstáculo de los necesarios contactos políticos.

En este marco, la cuestión del mérito, uno de los puntos nodales tanto del discurso académico como de los discursos oficiales, debe ser entendida desde el rol de las ideas y su complejidad. El mérito, como la eficiencia, es una idea neutra que debe ser cargada de sentido desde un discurso ideológico. El mérito podrá ser comprendido desde puntos tan subjetivos y diversos como la acumulación de titulaciones universitarias, la experiencia laboral anterior, el autodidactismo garantizado de alguna manera convincente, o los merecimientos por lealtad política. En el caso de Chile se ve que es importante tanto la capacidad individual como el complemento necesario de una buena trayectoria política.

\footnotetext{
24 Los directorios de las empresas públicas uruguayas están formado por cinco personas. Una norma no escrita es que los gobiernos dividan estos puestos en tres para el oficialismo y dos para la oposición. Está práctica se sedimentó históricamente entre blancos y colorados, que dominaron es espectro político del siglo XX.

25 Para ejemplos se instituciones informales e incumplimiento estricto de la ley en Uruguay se puede ver Oszlak (1972) y Panizza (2002).

26 Ferraro utiliza en su investigación la técnica de la entrevista con informantes clave que cuentan su experiencia directa, sus conocimientos, desde su posición de insider institucional. Para llegar a cada informante, se utiliza la técnica del snowballing (Yeager 2006: 913), por lo que siempre es un informante clave el que recomienda y genera el contacto con otro (Ferraro 2008: 111).
} 
Por último, subyace a esta situación una marcada tendencia a ocultar el verdadero funcionamiento de la política, negando de esta manera la posibilidad de que la población entienda las prácticas de gobierno reales. Esto se ve en la paradoja de que los políticos aceptan las instituciones informales como lo legítimo, pero no proceden a reacomodar la legislación pertinente -de modo de hacerla efectiva, útil y real- para formalizar la informalidad. Es decir, el imaginario político prefiere continuar desarrollando prácticas formalmente ilegales antes que reconocer públicamente un modo de hacer legítimo y naturalizado. En definitiva, lo legal en los países analizados no es un valor elemental uniforme en las prácticas políticas legítimas.

Sea como fuere, la administración pública chilena, evaluada a partir de índices técnicos, es una de las mejor conceptualizadas por la literatura académica. Por supuesto, la carencia de ética pública, si los índices económicos de un país son positivos, no pareciera ser un problema para los análisis y evaluaciones tecnocráticas. Quizás por ello, los países de la región no terminen por consolidad su desarrollo y lograr una administración verdaderamente efectiva que mejore la calidad de vida general.

\section{CONCLUSIONES}

Los discursos oficiales en torno a la reforma forman un conjunto narrativo significado fuertemente desde las ideas que construyen el imaginario político y determinan las maneras de entender la democracia, el Estado y lo político (Figura 1). Desde aquí, el modo de pensar e interpretar la coyuntura giró en torno al punto nodal central de incorporar lo económico como nexo de unión entre las distintas ideas, formando un metadiscurso de la política contemporánea. Las metas económicas significan por sí mismas y son, entonces, utilizadas como argumentos válidos para dar sentido general a planes de gobiernos particulares. Y, en este sentido, el discurso eminentemente técnico (presentado como una narrativa aideológica) fue el elegido para enunciar programas de acción.

A modo de cierre, se pueden sentenciar dos problemas graves de estos discursos que limitan la coherencia narrativa en torno a las reformas: su inexactitud semántica y su anclaje en una realidad simbólica exclusivamente institucional. 
Figura 1: Rol de los imaginarios políticos en la resignificación de la ambigüedad del discurso técnico

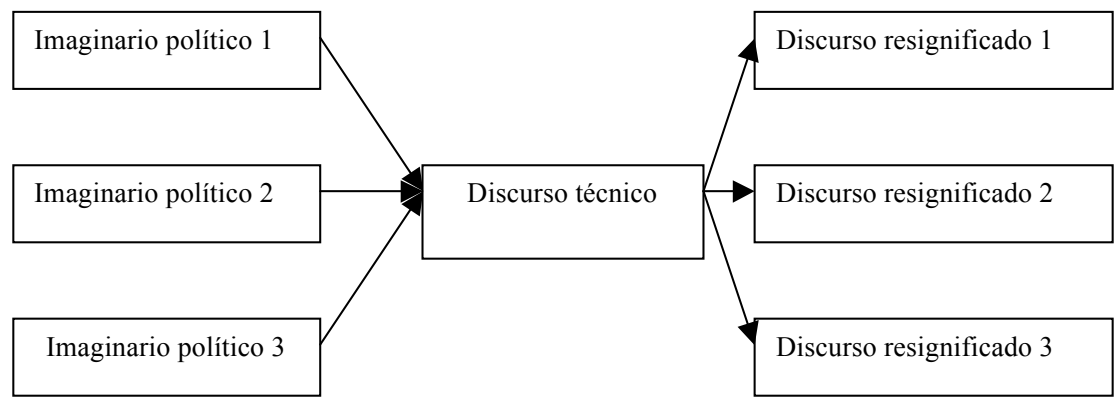

Fuente: Moriconi (2012: 305).

En primer lugar, la continuidad discursiva entre la literatura académica y los discursos oficiales se da en el marco de un conjunto de conceptos técnicos que no significan por sí mismos. Sin embargo, estos conceptos, como metas hacia dónde dirigirse, se han convertido en puntos nodales del discurso sobre las reformas, dando un matiz universalista a lo que debería ser y al cómo debería actuar una buena administración. Sin embargo, el estudio de las ideas rectoras que los resignifican ha sido pasado por alto en la literatura académica. Esta carencia es grave puesto que todo imaginario político, además de definir lo hacible y decible en un momento dado, parte necesariamente de un ideal de vida buena que determina lo que es justo dentro de cada sociedad (Panizza 2002). Estas consideraciones tienen una base axiomática arbitraria legitimada discursivamente, de modo que el cúmulo de ideas sobre el que se sustenta se instalará como marco hegemónico.

En segundo lugar, los diagnósticos discursivos y los programas de medidas no se refieren a una realidad simbólica pura o verdadera, sino que actúan sobre una realidad simbólica institucional determinada por el discurso de lo jurídico y lo legal. Las resignificaciones discursivas surgidas desde las instituciones informales no intervienen dentro de los análisis críticos que determinan los problemas de cada administración. De esta forma, los programas de mejora y modernización de las administraciones se enfocaron simplemente en modificaciones de las estructuras organizacionales y en nuevos marcos de motivación para las plantillas. Asimismo, sus sistemas de implementación parten de concepciones del poder y la política incompletas, por lo que resulta imposible estipular lógicas de sedimentación en una coyuntura que es mucho más compleja que la diagnosticada.

En definitiva, analizar el conflicto desde el rol de las ideas permite comprender que el problema no es, de ninguna manera, un simple conflicto técnico organizacional, y que las soluciones planteadas son, y serán, ineficientes si simplemente se basan en la construcción de nuevos conceptos técnicos impuros y en innovadores estructuras organizacionales. 


\section{REFERENCIAS}

Acuña, C. y Smith, W. (1994). The Political Economy of Structural Adjustment: The Logic of Support and Opposition to Neoliberal Reform. En Smith, W., Acuña, C. y Gamarra, EI (Eds.), Latin America's Political Economy in the Age of Neoliberal Reform. New Brunswick: Transaction.

Agor, W. (1971). The Chilean Senate: Internal Distribution of nfluence. Austin: University of Texas Press.

Aylwin, P. (1993). Mensaje Presidencial al Pleno del Congreso, 21 de Mayo, Presidencia, Santiago de Chile.

Bresser Pereira, L. (1996). Da Administraçao Pública Burocrática à Gerencial. Revista do Serviçio Público, 47 (1), 12-23.

Centro Latinoamericano de Administración para el Desarrollo (CLAD) (1998). Una Nueva Gestión Pública para América Latina. Caracas: Consejo Científico del CLAD.

Concertación de Partidos por la Democracia (1993). Un Gobierno para Nuevos Tiempos: Bases Programáticas del Segundo Gobierno de la Concertación. Santiago: Concertación de Partidos por la Democracia.

Cunill, N. (1997). Repensando lo Público a través de la Sociedad. Nuevas Formas de Gestión Pública y Representación Social. Caracas: CLAD, Editorial Nueva Sociedad.

Cypher, J. M. (2004). Pinochet Meets Polanyi? The Curious Case of the Chilean Embrace of "Free" Market Economics. Journal of Economic Issues, XXXVIII (2), $527-535$.

Domeniconi, H., Gaudio, R., y Guibert, A. (1992). Hacia un Estado Moderno: El Programa de Reforma Administrativa. Boletín Informativo Techint, 269, 3-28.

Domeniconi, H. (1996). La Reforma del Estado en la Argentina. Buenos Aires: Mimeo.

Fairclough, N. (1989). Language and Power. Londres y Nueva York: Longman. (1992). Discourse and Social Change. Cambridge: Polity Press.

Ferarro, A. (2006). Una Idea Muy Precaria: El Nuevo Servicio Civil y los Viejos Designados Políticos en Argentina. Latin American Research Review, 41 (2), 165-182.

(2007). Sobreviviendo a la Gobernanza, Ciencia de la Administración y Ciencia Política en el Estado Moderno, con Especial Referencia a Países de América Latina. Salamanca: Instituto Interuniversitario de Iberoamérica, Universidad de Salamanca. 
Imaginario Político y Discursos Técnicos en las Reformas Administrativas . (2008). Friends in High Places: Congressional Influence on the Bureaucracy in Chile. Latin American Politics and Society, 50 (2), 101- 129.

Foro Batllista (1994). El Uruguay entre Todos, Programa 2000. Montevideo: Foro Batllista.

Frei, E. (1994). Mensaje Presidencial al Pleno del Congreso, 21 de Mayo, Fundación Eduardo Frei, Centro de Documentación, Santiago de Chile.

-------. (1996). Discurso de Inauguración del Diálogo sobre Gobernabilidad Democrática, 2 de Septiembre, Fundación Eduardo Frei, Centro de Documentación, Santiago de Chile.

-------. (1998). Mensaje Presidencial al Pleno del Congreso, 21 de Mayo, Fundación Eduardo Frei, Centro de Documentación, Santiago de Chile.

Geddes, B. (1994). Politicians' Dilemma: Reforming the State in Latin America. Berkeley: University of California Press.

Ghio, J. M. y Etchemendi, S. (1998). Fugindo do Perigo: A Política da Reforma Administrativa na Argentina de Menem. Revista do Servico Público, 49 (2), 33-55.

Gil, F. (1966). The Political System of Chile. Boston: Houghton-Mifflin.

Habermas, J. (1973). Problemas de Legitimación en el Capitalismo Tardío. Buenos Aires: Amorrurtu.

Habermas, J. (1976). La Legitimidad Hoy. Revista de Occidente, 9, 3-12.

Haggard, S. y Kaufman, R. (1992). Institution and Economic Adjustment. En Haggard, S. y Kaufman, R. (Eds.), The Politics of Economic Adjustment. Princeton: Princeton University Press.

Hall, P. (1986). Governing the Economy. The Politics of State Intervention in Britain and France. Oxford: Oxford University Press.

Iacoviello, M. y Zuvanic, L. (2005). Desarrollo e Integración de la Gestión de Recursos Humanos en los Estados Latinoamericanos. Ponencia presentada en el X Congreso Internacional del CLAD sobre la Reforma del Estado y la Administración Pública, Octubre, Santiago de Chile.

Laclau, E. (2002). El Análisis Político del Discurso: Entre la Teoría de la Hegemonía y la Retórica. DeSignis, 2. Disponible en http://www.designisfels.net/ designis2_6.htm [25-4-2012].

Laclau, E. y Mouffle, C. (1985). Hegemony and Socialist Strategy. Verso: Londres. 
Menem, C. (1989). Discurso de Asunción frente a la Asamblea Legislativa, 8 de Julio. Presidencia de la Nación, Buenos Aires.

(1990a). Mensaje por Cadena Abierta de Radio y Televisión Exponiendo el Programa de Reforma del Estado y la Transformación Nacional, 1 de Agosto, Presidencia de la Nación, Buenos Aires.

(1990b). Acto de Privatización de Aerolineas Argentinas, 21 de Noviembre, Presidencia de la Nación, Buenos Aires.

(1990c). Discurso Durante la Entrega de Diplomas de Egresados a los Nuevos Administradores Gubernamentales, 26 de Diciembre, Presidencia de la Nación, Buenos Aires.

(1991a). Discurso Durante el Lanzamiento del SINAPA, 27 de Mayo, Presidencia de la Nación, Buenos Aires.

(1991b). Discurso Durante el Acto de Reencasillamiento del Personal del Ministerio de Salud y Acción Social al SINAPA, 7 de Noviembre, Presidencia de la Nación, Buenos Aires.

(1992). Discurso Durante el Acto de Inauguración de los Cursos de Alta Gerencia Pública, 19 de Mayo, Presidencia de la Nación, Buenos Aires.

Moriconi, M. (2012). Retórica Política y Administración Pública. Por Qué Fallan las Reformas Administrativas. México: CLACSO, Universidad Autónoma Metropolitana.

Moulián, T. (1997). Chile Actual: Anatomia de un Mito. Santiago: ARCIS-LOM.

North, D. (1990). Institutions, Institutional Change, and Economic Performance. Cambridge: Cambridge University Press.

Novaro, M. y Palermo V. (1996). Politica y Poder en el Gobierno de Menem. Buenos Aires: Grupo Editorial Norma.

O’Donnell, G. (1994). Delegative Democracy. Journal of Democracy, 5 (1), 13-23.

Oszlak, O. (1972). Diagnóstico de la Administración Pública Uruguaya. Nueva York: Naciones Unidas.

(1999). Quemar las Naves (o cómo Lograr Reformas Estatales Irreversibles). Ponencia presentada en el IV Congreso Internacional del CLAD sobre la Reforma del Estado y la Administración Pública, Noviembre, México.

--------.-. (2001). El Servicio Civil en América Latina y el Caribe: Situación y Retos Futuros. Ponencia presentada en el VI Congreso Internacional del CLAD sobre la Reforma del Estado y la Administración Pública, Noviembre, Buenos Aires. Panizza, F. (2002). Discurso e Instituciones en la Reforma de la Administración 
Imaginario Político y Discursos Técnicos en las Reformas Administrativas Pública Uruguaya. Revista Uruguaya de Ciencia Politica, 13, 59-93.

(2004). A Reform without Losers: The Symbolic Economy of Civil Service Reform in Uruguay. Latin American Politics and Society, 46 (3), 1-29.

Prats, J. (1999a). Del Clientelismo al Mérito en el Empleo Público, Análisis de un Cambio Institucional. Barcelona: Instituto Internacional de Gobernabilidad.

(1999b). Reforma del Estado y Cooperación para la Reforma del Estado en América Latina. Revista Nueva Sociedad, 160, 118-132.

Przeworski, A. (1991). Democracy and the Market: Political and Economic Reforms in Eastern Europe and Latin America. Cambridge: Cambridge University Press.

Rehren, A. (2000). Clientelismo Politico, Corrupción y Reforma del Estado en Chile. Santiago: Centro de Estudios Públicos.

Repetto, F. (2001). Causas y Azares de un Cambio Institucional Inconcluso: Historia Reciente de la Administración Pública Nacional en la Argentina. Ponencia presentada en el XVI Congreso Internacional del CLAD sobre la Reforma del Estado y la Administración Pública, Noviembre, Buenos Aires.

Sanguinetti, J. M. (1985). Discurso Presidencial al Asumir el Poder, 1 de Marzo, Presidencia, Montevideo.

(1995). Discurso Presidencial al Asumir el Poder, 1 de Marzo,

Presidencia, Montevideo.

Schick, A. (1998). Why Most Developing Countries Should Not Try New Zealand Reforms. The World Bank Research Observer, 13 (1), 123-131.

Scott, J. (1990). Domination and the Arts of Resistance, Hidden Transcripts. New Haven and London: Yale University Press.

Sexe, N. (2001). Diseño.com. Barcelona: Paidós.

Sigal S. y Verón, E. (1988). Perón o Muerte. Los Fundamentos Discursivos del Fenómeno Peronista. Buenos Aires: Hyspamérica.

Tapia-Videla, J. (1977). The Chilean Presidency in a Developmental Perspective. Journal of Interamerican Studies and World Affairs, 19 (4), 451-481.

Valenzuela, A. (1977). Political Brokers in Chile. Durham: Duke University Press. (1984). Parties, Politics, and the State in Chile: The Higher Civil Service. En Suleiman E. N. (Ed.), Bureaucrats and Policy Making: A Comparative Overview. New York: Holmes and Meier. 


\section{MORICONI}

Valenzuela, A. y Wilde, A. (1979). Presidential Politics and the decline of the Chilean Congress. En Smith J. y Musolf L. (Eds.), Legislatures in Development: Dynamics of Change in New and Old States. Durham: Duke University Press.

Waissbluth, M. (2006). La Reforma del Estado en Chile 1990-2005. De la Confrontación al Consenso. Santiago: Universidad de Chile, Facultad de Ciencias Físicas y Matemáticas.

Zizek, S. (1992). El Sublime Objeto de la Ideología. México: Siglo XXI.

Recibido: 06-07-2011

Aceptación de la versión final: 03-05-2012 